\title{
Increasing the Speed of EELS/EDS Mapping Through Dynamic/Adaptive Sampling Methodologies
}

Karl A. Hujsak ${ }^{1}$, Andrew Stevens ${ }^{2,3}$, Libor Kovarik ${ }^{4}$, Andrey Liyu ${ }^{4}$, Nigel D. Browning ${ }^{4,5}$, and Vinayak P. Dravid ${ }^{1,6}$

1. Department of Materials Science \& Engineering, Northwestern University, Evanston, IL.

2. OptimalSensing, Southlake, TX.

3. Department of Electrical and Computer Engineering, Duke University, Durham, NC.

4. Pacific Northwest National Laboratory, Richland, WA.

5. Department of Materials Engineering, University of Liverpool, Liverpool, United Kingdom

6. Electron Probe Instrumentation Center (EPIC) Facility, NUANCE Center, Northwestern University, Evanston, IL.

Next generation electron microscopes equipped with aberration correctors and sensitive detectors have enabled routine collection of atomic-scale information with sufficient signal-to-noise ratio from a variety of technologically relevant materials. In the case of Scanning Transmission Electron Microscopy (STEM), the ability to focus a large electron flux into a picometer probe has also benefitted the collection of correlative spectral information, primarily in the forms of Electron Energy Loss Spectroscopy (EELS) and Energy Dispersive X-ray Spectrometry (EDS). Collection of atomic scale EELS and EDS spectra over a grid of pixels, referred to as a spectrum image, is now routinely achievable outside of specialized labs.

However, the large inelastic background component in EELS and the limited available detector crosssections in EDS means that each pixel of the spectrum image requires a large integrated dwell time (on the order of milli-seconds to seconds). This results in a relatively poor dose-efficiency, where the material must be subjected to a large incident flux for a comparatively small signal. For atomically resolved spectrum images, there are a limited number of materials with sufficient radiation hardness to withstand such a damaging measurement, as each atomic column may often be subject to multiple integrated acquisitions. Many materials of interest often gain often gain their structure from a complex arrangement of weaker bonds between a hybrid set of metal and organic elements (metal/covalent organic frameworks), which are easily altered due to radiolysis. The long dwell times per pixel also mean total acquisition times for large maps can stretch from hours, to even days. Ensuring a sufficient field of view (FOV) such that the atomic level information is truly representative of the overall material structure is challenging, and often a compromise must be made between sample stability, final resolution, and FOV.

Random sub-sampling has been proposed to both reduce the integrated area dose and time-to-collect highresolution images in Scanning Electron Microscopy (SEM) [1]. Observing that not all pixels in an image contribute meaningful information or contrast, the missing pixels can be restored off-line by exploiting statistical correlations between neighbors. Using a random mask assumes important pixels are also randomly distributed, which is generally not true. Thus, many sub-sampling techniques are limited by their 'static' masks and cannot obtain sampling percentages lower than $15-20 \%$.

Approaches which can tune the mask of selected pixels 'on-the-fly', referred to as adaptive or dynamic sampling, have recently been shown to achieve state-of-the-art image quality at extremely low sampling rates $[2,3]$. Such algorithms follow a general approach as follows: collect an initial set of measurements 
randomly, perform a crude approximation of the sample structure, select the next single (or batch) of measurements which is expected to yield the maximum amount of information, perform the measurements, and iterate. With modern computation such selection processes can be performed on timescales orders of magnitude lower than the time to collect spectra with sufficient signal-to-noise ratio.

We will discuss two implementations of dynamic, or adaptive, sampling strategies in electron microscopy, based on different forms of prior information. First, by Fourier filtering heavily under sampled images of periodic lattices, guesses about the centroid positions of the various atomic columns in a region of interest can be made. By taking several frames with extremely low sampling percentages where the dose is allocated to the expected positions of those centroids, an atomically resolved image can be constructed at significantly lower doses than conventional imaging [2]. Next, we will discuss a general framework for performing dynamic sampling on arbitrary periodic or non-periodic samples based on mapping descriptors in an under-sampled image to expected utility [3]. An experimental framework for performing dynamic sampling on arbitrary STEMs will be presented, as well as a discussion of the prior information encoded in the use of training images to construct a mapping between descriptors and utility. Finally, we will discuss the trade-off between exploration and exploitation at ultra-low sampling rates, where there is not a sufficient dose to both search for new areas of interest and/or further improving the reconstruction quality of previously identified structures [4].

\section{References:}

[1] K. Hujsak et al, M\&M (2016), p. 1.

[2] A. Stevens et al, (2018). Applied Physics Letters 112(4) (2018), p. 043104.

[3] G.M. Godaliyadda et al, Electronic Imaging 201619 (2016), p. 1.

[4] This material is based on research sponsored by the Air Force Research laboratory under agreement

No. FA8650-15-2-5518 and Air Force Office of Scientific Research under Award No. FA9550- 12-1-0280.

This work made use of the EPIC facility of Northwestern University's NUANCE Center, which has received support from the Soft and Hybrid Nanotechnology Experimental (SHyNE) Resource (NSF ECCS-1542205); the MRSEC program (NSF DMR-1720139) at the Materials Research Center; the International Institute for Nanotechnology (IIN); the Keck Foundation; and the State of Illinois.

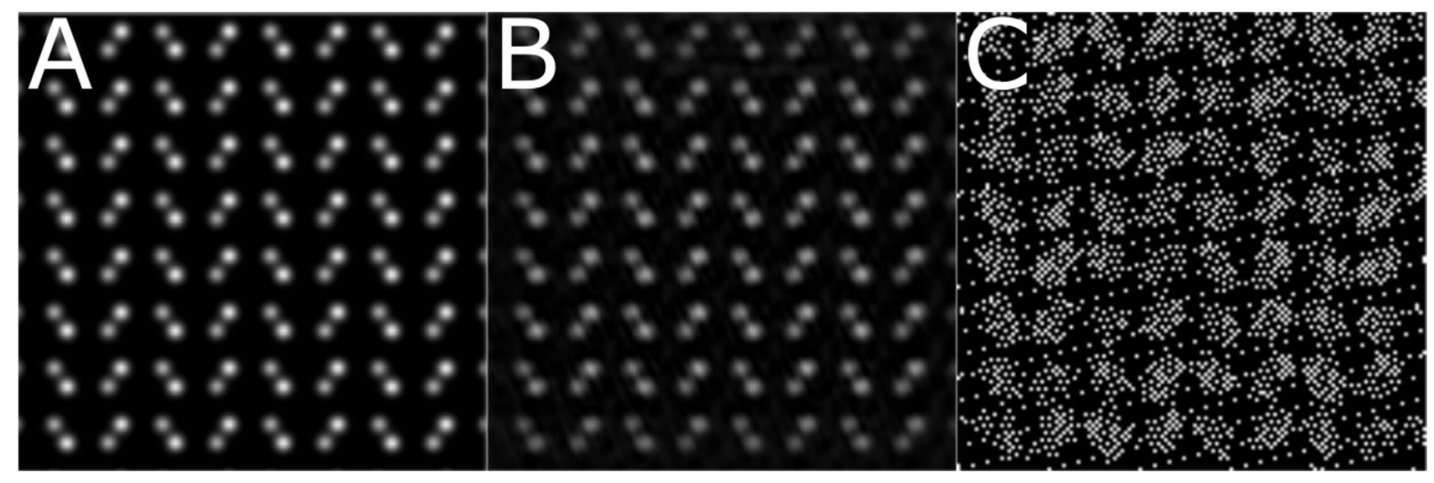

Figure 1. A. Simulated ZnSe HAADF image from Stevens, et al. [2] B. Reconstruction after a simulated dynamic sampling experiment, where each unsampled pixel was assigned the mean value of its five nearest neighbors. During the simulation, no explicit or implicit expressions of periodicity are assumed to guide the algorithm. For final presentation, the image was Fourier filtered. C. The final mask of points after $12 \%$ sampling using the dynamic sampling approach of Godaliyadda, et al. [3]. Note the prioritization of lattice positions over other potential measurements. 